\section{The partial Talbot effect and its use in measuring the coherence of synchrotron X-rays}

\section{Jean-Pierre Guigay, ${ }^{a, b *}$ Simon Zabler, ${ }^{a}$ Peter Cloetens, ${ }^{a}$ Christian David, ${ }^{\mathrm{c}}$ Rajmund Mokso ${ }^{\mathrm{a}}$ and Michel Schlenker ${ }^{b}$}

\author{
${ }^{a}$ European Synchrotron Radiation Facility, BP 220, F-38043 \\ Grenoble, France, 'baboratoire Louis Néel du CNRS, BP 166, \\ F-38042 Grenoble, France, and ${ }^{C}$ Paul Scherrer Institut, CH-5232 \\ Villigen, Switzerland. E-mail: guigay@esrf.fr
}

The Talbot effect is the self-imaging, at distances $D$ multiple of $D_{\mathrm{R}}$, of the intensity downstream of a periodic object. Earlier work with hard synchrotron radiation $\mathrm{X}$-rays showed the variation with $D$ of the fundamental Fourier component of intensity to be a good measurement of beam coherence. Any higher-order Fourier coefficients $\tilde{I}(D, m>1)$ would be periodic with a reduced period $D_{\mathrm{R} m}=D_{\mathrm{R}} / m$ for an ideally coherent incident beam (partial Talbot effect). The degree of coherence $\gamma(x)$ is sampled through the ratio of $\tilde{I}(D, m)$ at $D=0$ and multiples of $D_{\mathrm{R} m}$. This requires the Fourier coefficient for $D=0$, which is not accessible for a phase object (no contrast at $D=0$ ). However, the ratio of the slopes of $\tilde{I}(D, m)$ at $D=0$ and $D=p D_{\mathrm{R} m}$ also provides this information. Furthermore, a characterization of $\gamma(x)$ is possible, provided an assumption is made on its shape, using only the ratio of the Fourier coefficient $\tilde{I}(D, m)$ of two images a distance $p D_{\mathrm{R} m}$ apart. Experiments with one- and two-dimensional phase gratings and a mixed (amplitude and phase) two-dimensional grating confirm that the partial Talbot effect approach is viable. It requires a reduced range of distances, and yields important results directly, obviating the need for computer fits. In particular, $8 \%$ of the beam intensity was found to have very low coherence in the vertical direction, probably due to monochromator imperfection.

Keywords: X-ray optics; Talbot effect; coherence measurements.

\section{Introduction}

The Talbot effect (Talbot, 1836) is a long-familiar spectacular consequence of Fresnel diffraction, itself a description of the effect of optical propagation over a finite distance. It consists of the lensless replication, at positions periodically distributed along the propagation direction, of the optical amplitude distribution due to a one- or two-dimensionally periodic object illuminated by a coherent wave (Winthrop \& Worthington, 1965; Patorski, 1989). This self-imaging effect was explored in the case of hard X-rays from a long beamline (ID19 at ESRF), expected to feature high spatial coherence, at a third-generation synchrotron radiation source (Cloetens et al., 1997). It was then found, using a one-dimensional phase grating, that the variation with defocusing distance $D$ of the first Fourier coefficient of the intensity distribution provides a way of measuring the degree of spatial coherence of the X-ray beam, and hence the source size. This approach is particularly robust because it is independent of the shape of the periodic object and the transfer function of the detector. In this paper, we investigate extensions of this approach, and use them to determine the source size in the case of a beamline with substantially lower coherence than in the initial measurement. This study handles several points in order to make the coherence measurement as practical as possible: the choice of the object type (phase or/and amplitude object; one- or two-dimensional grating), the choice of the order $m$ of the Fourier coefficient, and the choice of the distances.

The effect of Fresnel diffraction can be described, in the case of a one-dimensional sample which we will retain for simplicity's sake in most of the discussion below, by convolving the amplitude distribution $g(x)$ right after the object, with the propagator $\exp \left(i \pi x^{2} / \lambda D\right)$, where $\lambda$ is the radiation wavelength. In terms of Fourier transforms, $f$ being the spatial frequency conjugate to the coordinate $x$, the Fourier transform of the amplitude distribution, $\tilde{g}(f)$, is multiplied by $\exp \left(-i \pi \lambda D f^{2}\right)$ (Goodman, 1968). This term is equal to unity for distances $D=p D_{\mathrm{T}}$, with $p$ an integer and $D_{\mathrm{T}}$ the Talbot distance, $D_{\mathrm{T}}=2 a^{2} / \lambda$ for any periodic object with period $a$, because $\tilde{g}(f)$ is then non-zero only for $f=m / a$ with $m$ an integer. The effective condition for perfect replication can actually be taken as $D=p D_{\mathrm{R}}$ with $p$ an integer and $D_{\mathrm{R}}=D_{\mathrm{T}} / 2$ because, for odd multiples of $D_{\mathrm{R}}$, the Fourier component of order $m$ of the amplitude distribution is multiplied by $(-1)^{m}$. The resulting amplitude distribution, being just shifted by $a / 2$, is in practice undistinguishable from the original. We will call $D_{\mathrm{R}}=a^{2} / \lambda$ the replication distance.

In this paper, we discuss properties of the Fourier coefficients of the intensity distribution in the Fresnel diffraction pattern of a periodic object and use those properties to measure in a simple way the degree of coherence on a bending-magnet beamline. We show, by introducing the partial Talbot effect, that the variation with defocusing distance $D$ of the higher Fourier coefficients of the intensity would be periodic, with periods smaller than $D_{\mathrm{R}}$, for completely coherent illumination. Their actual damped behaviour can thus provide quantitative information about the beam coherence directly, from a smaller range of defocusing distances than using the standard Talbot effect.

The amplitude distribution just after the specimen, $g(x)$, is simply proportional to the amplitude transmission function of the sample if the incident wave is plane. In the case of a cylindrical (or spherical) incident wave, emitted from a point source at finite distance $L$ from the object, $g(x)$ contains a non-periodic factor $\exp \left(i \pi x^{2} / \lambda L\right)$ : a simple change of variable in the Fresnel diffraction integral shows that selfimaging is then produced at modified distances $n L D_{\mathrm{R}} /\left(L-n D_{\mathrm{R}}\right)$ with $n$ being an integer. More generally, the diffraction pattern which would be observed at distance $D$ from the sample in the case of an infinitely distant source is actually produced at the distance $D^{\prime}=$ $D /(1-D / L)$. We will base the descriptions on $D$, the defocusing distance, rather than on $D^{\prime}$, the actual specimen-detector distance.

\section{The partial Talbot effect}

The Fourier transform of the intensity distribution (intensity spectrum) $I_{D}(x)$ of a Fresnel diffraction pattern can be expressed, if the incident beam is considered as a quasi-monochromatic ensemble of mutually incoherent plane waves, by the formula (Guigay, 1978; Cloetens, 1999)

$$
\tilde{I}_{D}(f)=\gamma(\lambda D f) \exp \left(-i \pi \lambda D f^{2}\right) \int_{-\infty}^{\infty} \mathrm{d} \eta \exp (-i 2 \pi \eta f) g(\eta) g^{*}(\eta+\lambda D f),
$$

where $\gamma(\lambda D f)$ is the spatial degree of coherence between two points in the object plane a distance $\lambda D f$ apart. $\gamma(\lambda D f)$ is unity for perfect coherence and, by definition, whatever the coherence, for $D=0$. According to the Van Cittert-Zernike theorem (Born \& Wolf, 1999), $\gamma(\lambda D f)$ is related to the normalized angular distribution $S(\theta)$ of the incident beam by a Fourier transformation 


$$
\gamma(\lambda D f)=\int \mathrm{d} \theta \exp (i 2 \pi D f \theta) S(\theta)=\tilde{S}(D f) .
$$

If $g(x)$ is a periodic function with period $a$, the intensity distribution $I_{D}(x)$ at distance $D$ is itself periodic in $x$, with the same period $a$, and can be expanded into a Fourier series with coefficients $\tilde{I}(D, m)(m$ integer), which are, according to (1),

$$
\begin{aligned}
\tilde{I}(D, m)= & \gamma(\lambda D m / a) \exp \left(-i \pi \lambda D m^{2} / a^{2}\right) a^{-1} \\
& \times \int_{0}^{a} \mathrm{~d} \eta \exp [-i 2 \pi \eta m / a] g(\eta) g^{*}(\eta+m \lambda D / a) .
\end{aligned}
$$

The properties of these coefficients, as functions of the defocusing distance $D$, are the subject of the present discussion.

Let us first consider the ideal case of perfectly coherent illumination, for which $\gamma(\lambda D m / a)=1$. Then $\tilde{I}(D, m)$ is clearly, apart from a factor $(-1)^{m}$, a periodic function of $D$, with period $D_{\mathrm{R} m}=a^{2} / m \lambda$. For $D=p a^{2} / \lambda$, all the Fourier coefficients are identical for all integral values of $p$, to within the change in sign $(-1)^{m}$ : this is the usual or full Talbot effect. At distances $D=p\left(a^{2} / \lambda m\right)=p D_{\mathrm{R} m}$, only those Fourier coefficients whose order is a multiple of $m$ are unchanged for different values of $p$, to within a change in sign $(-1)^{m p}$. We suggest calling this partial preservation of the intensity spectrum the partial Talbot effect.

This partial Talbot effect is closely related to the so-called fractional Talbot diffraction, which deals with Fresnel diffraction by periodic objects at distances equal to fractions of the Talbot distance $D_{\mathrm{T}}$ and which is used for some applications in visible-light optics (Testorf \& Ojeda-Castañeda, 1996; Zhou et al., 1998; Arrizon \& RojoVeláquez, 2001). At such distances, $D=D_{\mathrm{T}}(p / q)$, where $p$ and $q$ are relative prime integers, the amplitude distribution $g_{D}(x)$ at distance $D$ can be expressed from the values of the object function $g\left(x^{\prime}\right)$ for $x^{\prime}=x+l a / q$, with $l$ an integer running from 0 to $q-1$. This means that the Fresnel diffraction integral is simplified into a sum of $q$ terms, this sum being actually reduced to $q / 2$ terms if $q$ is even. For instance, we obtain $g_{D}(x)=g(x+a / 2)$ for $p / q=1 / 2$, and we obtain $g_{D}(x)=$ $[g(x)+i g(x+a / 2)] / 2^{1 / 2}$ for $p / q=1 / 4$ (Guigay, 1971; Arrizon \& OjedaCastañeda, 1992).

In the following, we will be concerned with the particular case of a binary two-dimensional phase grating $g(x, y)=h(x) h(y)$, where $h(x)$ is a periodic binary function of period $a$ such that $h(x)=\exp (i \Phi)$ for $0<x<w a$ and $h(x)=1$ for $w a<x<a$, with $w<1 / 2$. It is then possible to perform the integration of (2) generalized to the two-dimensional case and find along the $x$-direction,

$$
\begin{aligned}
\tilde{I}(D, m)= & \gamma\left(a D / D_{\mathrm{R} m}\right) \exp (-i \pi w m) \sin \left(\pi m D / D_{\mathrm{R} m}\right) \\
& \times[\sin \Phi \sin (\pi w m) \pm(\cos \Phi-1) \cos (\pi w m)] / \pi m,
\end{aligned}
$$

with + for $D<w D_{\mathrm{R} m}$ and - for $(1-w) D_{\mathrm{R} m}<D<D_{\mathrm{R} m}$, and

$$
\begin{aligned}
\tilde{I}(D, m)= & \gamma\left(a D / D_{\mathrm{R} m}\right) \exp (-i \pi w m) \sin (\pi w m)\left[\sin \Phi \sin \left(\pi m D / D_{\mathrm{R} m}\right)\right. \\
& \left.+(\cos \Phi-1) \cos \left(\pi m D / D_{\mathrm{R} m}\right)\right] / \pi m
\end{aligned}
$$

for $w D_{\mathrm{R} m}<D<(1-w) D_{\mathrm{R} m}$.

\section{Application to coherence measurements}

When the incident beam is not perfectly coherent, the periodicity as a function of distance will be disrupted. The variation with $D$ of the Fourier coefficients of the Fresnel-diffracted intensity makes it possible to quantitatively determine the coherence width in the object plane, or the angular source size. Formula (3) written for $D=$ $p D_{\mathrm{R} m}$ shows that

$$
\tilde{I}\left(p D_{\mathrm{R} m}, m\right)=\gamma(p a)(-1)^{m p} \tilde{I}(0, m)=\tilde{S}(p a / \lambda)(-1)^{m p} \tilde{I}(0, m) .
$$

Thus we obtain the degree of coherence $\gamma(p a)$ from the ratio $r(0, p, m)$ of the Fourier coefficient of order $m$ at the distances $D=$ $p D_{\mathrm{R} m}$ and $D=0$. By varying $p, \gamma(x)$ can be sampled, with the period $a$ of the specimen. It may be noted that the use of higher harmonics $(m>1)$ does not mean that the coherence function is sampled at smaller intervals $a / m$, but it provides the important experimental advantage to use distances that are $m$ times smaller.

Obtaining $\gamma(p a)$ by this approach is not possible with a pure phase grating since $\tilde{I}(0, m)=0$ for any $m \neq 0$ in this case. The most straightforward way around the problem of this lack of contrast at $D=0$ is to use a mixed (amplitude and phase) grating instead of a pure phase grating. However, we found another possibility: measuring the derivative $\tilde{I}^{\prime}(D, m)$ with respect to the distance $D$ of the Fourier coefficient of order $m$. It is readily seen that relation (6) is valid for the derivatives too,

$$
\tilde{\boldsymbol{I}}^{\prime}\left(p D_{\mathrm{R} m}, m\right)=\gamma(p a)(-1)^{m p} \tilde{\boldsymbol{I}}^{\prime}(0, m),
$$

under the condition that $\tilde{I}(0, m)=0$, which is precisely satisfied in the case of a phase grating. Relation (7) is also approximately valid if $\lambda(m / a)\left|\gamma^{\prime}(p a) \tilde{I}(0)\right|<<\left|\gamma(p a) \tilde{I}^{\prime}(0)\right|$.

The derivative $\tilde{I}^{\prime}(0, m)$ is generally not equal to zero, particularly in the case of a phase grating; its value can be determined from images recorded with a small increment in distance $D$. In this paper, we do not make optimal use of this possibility, because we realised this after the experiments and therefore our choice of experimental distances did not incorporate this optimally. We can, however, test the validity of this new approach, which substantially extends the possibilities of the Talbot effect for coherence measurements, especially with phase gratings.

In the case of a phase grating, another useful possibility is to use the ratio

$$
\begin{aligned}
r\left(D_{0}, p, m\right) & =\left|\tilde{I}\left(D_{0}, m\right) / \tilde{I}\left(D_{0}+p D_{\mathrm{R} m}, m\right)\right| \\
& =\left|\gamma\left(\lambda D_{0} m / a\right) / \gamma\left(\lambda D_{0} m / a+p a\right)\right|
\end{aligned}
$$

of the Fourier coefficient of order $m$, at some non-zero distance $D_{0}$ and at $D_{0}+p D_{\mathrm{R} m}$. Determining the variation of $\gamma(x)$ from this ratio requires an a priori assumption on the form of this function, or equivalently on the angular intensity distribution $S(\theta)$ of the X-ray beam, as was performed by Cloetens et al. (1997) in the case $m=1$, $p=1, D_{0}=D_{\mathrm{R}} / 2$. If the incident beam is assumed to have a Gaussian angular distribution described by $S(\theta)=\exp \left[-(2 \theta / W)^{2} \ln 2\right]$, where $W$ is the FWHM angular source size of the X-ray beam, the degree of coherence is, according to expression (2),

$$
\gamma(x)=\exp \left[-(\pi W x / \lambda)^{2} / 4 \ln 2\right]=\exp \left[-(W x / 0.530 \lambda)^{2}\right] .
$$

From (8), we obtain

$$
W^{2}=\left(4 \ln 2 / \pi^{2}\right) \ln \left[r\left(D_{0}, p, m\right)\right] /\left[(p a / \lambda)^{2}+2 m p D_{0} / \lambda\right] .
$$

The transverse coherence width $l_{\mathrm{t}}$ can be defined such that $\gamma\left(l_{\mathrm{t}}\right)=1 / 2$ and is then equal to $(2 \ln 2 / \pi) \lambda / W \simeq 0.44 \lambda / W$.

Further, under the assumption of Gaussian distributions and if the degree of coherence $\gamma(x)$ is known for a given value $x$, the angular source size $W$ may be obtained from

$$
\begin{aligned}
W & =\left[2(\ln 2)^{1 / 2} / \pi\right](\lambda / x)\left\{\ln \left[\gamma^{-1}(x)\right]\right\}^{1 / 2} \\
& =0.530(\lambda / x)\left\{\ln \left[\gamma^{-1}(x)\right]\right\}^{1 / 2} .
\end{aligned}
$$

The size of the source is obtained by multiplying the angular source size $W$ by the source-to-specimen distance $L$. 


\section{Experimental approach, samples and results}

The primary data in the present investigation consist of sets of images of the periodic specimen, illuminated by hard $(17.5 \mathrm{keV}, \lambda=70.7 \mathrm{pm})$ $\mathrm{X}$-rays produced by the bending magnet of the optics beamline BM05 of the European Synchrotron Radiation Facility, monochromated by a double perfect silicon monochromator using the 111 reflection in symmetrical Bragg geometry, in the vertical plane. The experiments were performed at a distance $L=55 \mathrm{~m}$ from the source with the synchrotron ring operating in the uniform mode, at an electron current of about $180 \mathrm{~mA}$.

The images were recorded using an X-ray-sensitive converter, coupled via a visible-light microscope-type optical set-up to a cooled CCD FReLoN camera (Koch et al., 1998, 2000), set for an effective pixel size of $0.7 \mu \mathrm{m}$.

The specimen-detector distance was systematically varied using an accurate translation stage for distances $D^{\prime}<0.82 \mathrm{~m}$, with the stage moved away from the specimen for larger distances. 'Dark' images (without X-ray beam) as well as images without the specimen were also recorded to perform 'flat-field' corrections for inhomogeneity of the beam, owing to the monochromator and windows upstream, and detector response. The images were then submitted to a fast one- or two-dimensional Fourier transform procedure, yielding the first (orders $m$ from 1 to 3 ) Fourier coefficients $\tilde{I}(D, m)$ as a function of propagation or defocusing distance $D$. In practice, only the modulus of the Fourier coefficients will be considered.

The first object used was a one-dimensional optical grating replica (Hilger \& Watts Student Grating). It had an approximately sineshaped profile, with a period of $6.35 \mu \mathrm{m}$ and a peak-to-peak height modulation of $1.1 \mu \mathrm{m}$. The other phase object we used, an etched pattern on silicon, was two-dimensional: it had a binary square profile, with period along two perpendicular directions of $8.0 \mu \mathrm{m}$ and peak-to-valley height difference of $2.9 \mu \mathrm{m}$, corresponding to a phase shift of $0.41 \mathrm{rad}$ at a photon energy of $17.5 \mathrm{keV}$. The widths of the regions with the two values of thickness were approximately equal. A scanning electron microscopy image of this silicon grating is shown in Fig. 1. We also used, as a mixed (phase and attenuation) grating, a square gold grid for electron microscopy (Agar G2786A 2000 mesh gold), with the bars $4 \mu \mathrm{m}$ wide and $5.1 \mu \mathrm{m}$ thick. The period along the two directions parallel to the bars was $12.5 \mu \mathrm{m}$. The intensity attenuation factor $\exp (-\mu t)$ for the metal part was then 0.35 for $17.5 \mathrm{keV}$ photons, with a phase shift of $7.35 \times 2 \pi \mathrm{rad}$.

Two-dimensional gratings with square phase, and possibly attenuation, modulations give the possibility of exploring three aspects. It becomes possible to gain simultaneously information on

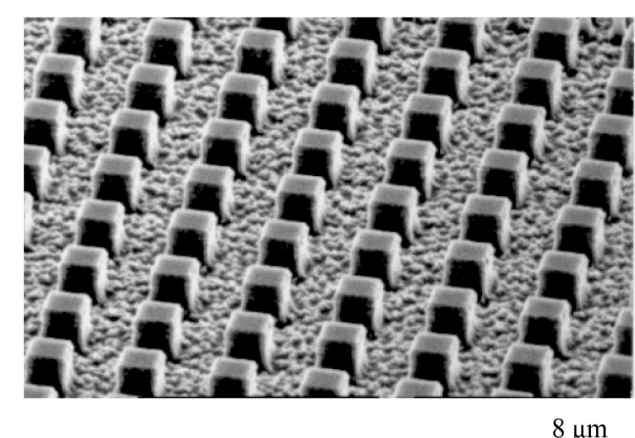

$8 \mu \mathrm{m}$

Figure 1

Scanning electron micrograph of silicon structures generated by electronbeam lithography and reactive ion etching used as a two-dimensional phase grating. the vertical and horizontal coherence widths, and hence source sizes. Square modulation implies that there are several strong harmonics in the Fourier expansion of the intensity, making it possible to draw information from more than one Fourier coefficient. The presence of attenuation could provide useful supplementary data in comparison with phase-only situations.

The images for the one-dimensional phase grating with sineshaped corrugation, $6.35 \mu \mathrm{m}$ period, are similar to the results from the same grating measured by Cloetens et al. (1997) on ID19, a beamline at the ESRF where the beam has larger coherence widths. The higher harmonics are very weak in this case, because the phase modulation is small and the thickness variation is essentially sinusoidal. Using the approach based on the full Talbot effect, the horizontal FWHM of the source intensity distribution, obtained with the one-dimensional phase grating, with its lines vertical, is 269 (8) $\mu \mathrm{m}$. The same measurement performed with the grating turned by $90^{\circ}$ yields, for the vertical FWHM, a value of $79(8) \mu \mathrm{m}$. The corresponding angular source sizes are $W_{\mathrm{H}}=4.90 \mu \mathrm{rad}$ and $W_{\mathrm{V}}=$ $1.40 \mu \mathrm{rad}$.

Fig. 2 shows the images obtained at $D \simeq 0, D=D_{\mathrm{R}} / 2, D=D_{\mathrm{R}}=$ $0.90 \mathrm{~m}$ and $D=3 D_{\mathrm{R}} / 2$ for the two-dimensional square phase grating, with periods in perpendicular directions of $8.0 \mu \mathrm{m}$. The appearance, disappearance and reappearance of contrast is obvious, as is the decrease in maximum contrast with increasing $D$, a consequence of the finite coherence width of the beam, and therefore of the non-zero size of the source. The decrease in contrast is seen to be more severe in the horizontal direction, pointing qualitatively to the anisotropy of the source. This is quantitatively shown in Fig. 3, where the variation with $D$ of the absolute values of the first, second and third Fourier coefficients of the intensity are shown. Fig. 3 shows, along with the
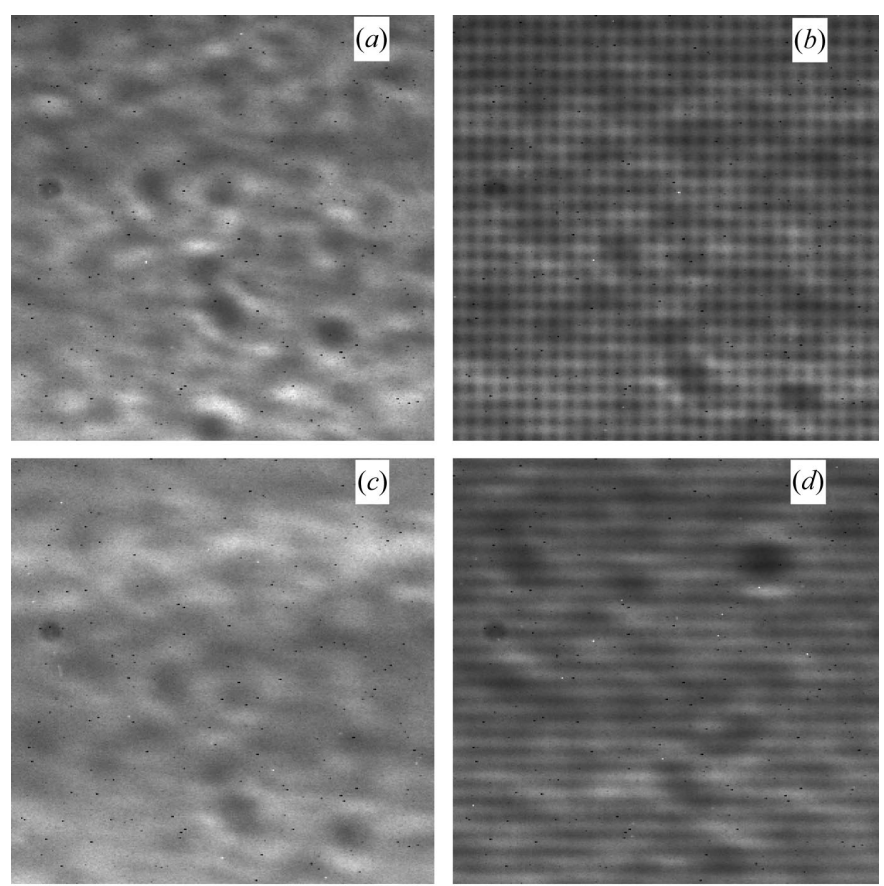

$50 \mu \mathrm{m}$

Figure 2

Images of a two-dimensional phase grating with period of $8.0 \mu \mathrm{m}$, with approximately equal widths for the thicker and the thinner regions, at defocusing distances of (a) $D=8 \mathrm{~mm},(b) D=D_{\mathrm{R}} / 2$, (c) $D=D_{\mathrm{R}}$ and (d) $D=$ $3 D_{\mathrm{R}} / 2$. Throughout this paper, the photon energy used is $17.5 \mathrm{keV}$, corresponding to wavelength $\lambda=70.7 \mathrm{pm}$; hence $D_{\mathrm{R}}=905 \mathrm{~mm}$. 

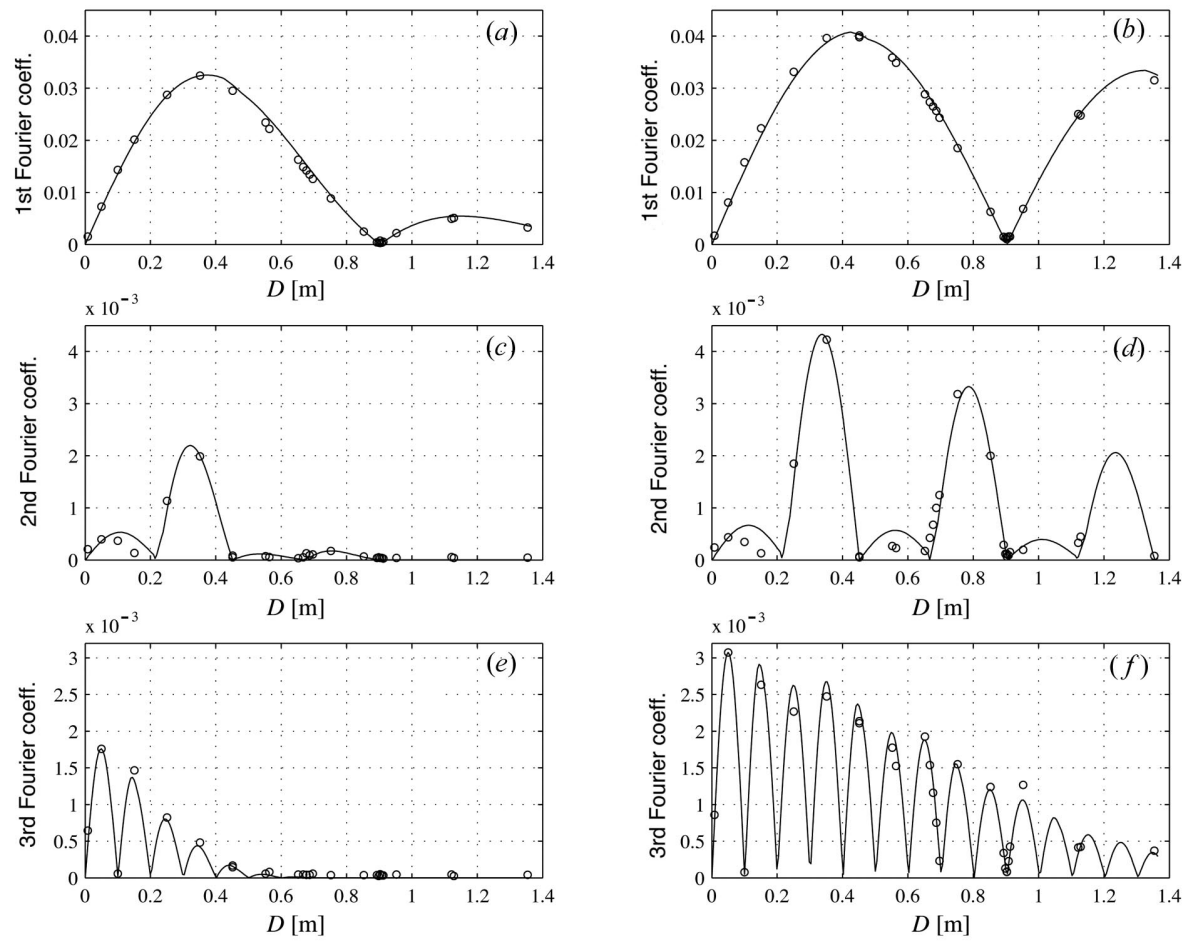

Figure 3

Variation with $D$ of the fundamental $(m=1)$, the second $(m=2)$ and the third harmonic $(m=3)$ of the variation along the $(a),(c),(e)$ horizontal and $(b),(d),(f)$ vertical directions, for the two-dimensional phase grating with period $8 \mu \mathrm{m}$. The circles correspond to the experiments, whereas the solid lines correspond to simulations. Best fits were obtained assuming a ratio $w$ of the binary grating equal to 0.465 . The simulations include a correction factor taking into account the decrease of the detector sensitivity when the spatial frequency under consideration is increased. This correction factor was adjusted such that the simulation agrees with the experimental data point with the highest value. The remaining discrepancies between the simulations and measurements may be attributed partly to imperfections in the modeling of the grating.

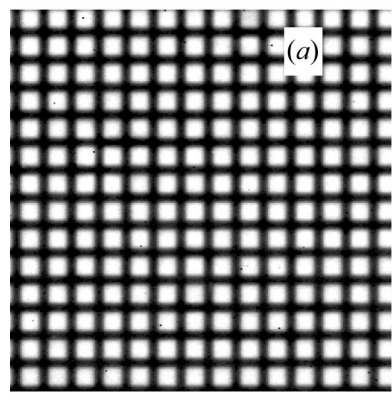

Figure 4

Images of the two-dimensional mixed (amplitude and phase) object, a gold grid for transmission electron microscopy with period $a=12.5 \mu \mathrm{m}$ for defocusing distances $(a) D=5 \mathrm{~mm},(b) D=547 \mathrm{~mm}$ and $(c) D=$ $2.2 \mathrm{~m}=D_{\mathrm{R}}$.

experimental points, curves calculated using values of $79 \mu \mathrm{m}$ for the vertical and $269 \mu \mathrm{m}$ for the horizontal source sizes. It is seen that some of the measured points are near a maximum of the third Fourier coefficient, and not near a zero. These points cannot give the slope directly. Consequently, for the slope method, we had to introduce a correction assuming a sinusoidal variation for the third coefficient as a function of $D$, as expected from equation (4) for this phase grating.

Fig. 4 presents images of a mixed phase and amplitude object, the gold grid for transmission electron microscopy, obtained at $D=5 \mathrm{~mm}$, $D=547 \mathrm{~mm}$ and $D=D_{\mathrm{R}}=2.2 \mathrm{~m}$. Fig. 5 shows the first and second
Fourier coefficients of the images of the grid. Here, in spite of the fact that it is a mixed grating, the third harmonic of the intensity is very small for $D \simeq 0$, because the bar width is close to one-third of the period.

The coherence properties can also be obtained through the partial Talbot effect, using either the square-profile phase grating or the mixed object (gold grid for transmission electron microscopy). As discussed above, the quantity obtained directly using expressions (6) or (7) is the degree of coherence between points that are a distance $x=p a$ apart, either in the vertical direction, $\gamma_{\mathrm{V}}(x)$, or in the horizontal direction, $\gamma_{\mathrm{H}}(x)$. The corresponding angular source sizes $W_{\mathrm{V}}$ and $W_{\mathrm{H}}$ are calculated using (11). The experimental results are summarized in Table 1 . The column titled 'Approach' indicates whether the data are obtained from the ratio $r(0, p, m)$ of the Fourier coefficients at $D=0$ and $D=p D_{\mathrm{R} m}$ [' $r(0, p, m)$ '; expression (6)], from the ratio of their slopes at $D=0$ and $D=p D_{\mathrm{R} m}$ ['slopes'; expression (7)], or from the ratio of the Fourier coefficients at $D=D_{0}$ and $D=D_{0}+p D_{\mathrm{R} m}\left[{ }^{\prime} r\left(D_{0}, p, m\right)\right.$ '; expression (10)]. The measurements in Table 1 are condensed in Fig. 6 to profiles of the degree of coherence as a function of distance both in the vertical and horizontal directions. The dots correspond to the discrete measurements, whereas the full lines correspond to fits weighted according to the error bars of the experimental data points. In the horizontal direction a Gaussian fit as given by expression (9) is used with an angular source size $W_{\mathrm{H}}=4.98$ (6) $\mu \mathrm{rad}$. In the vertical direction, however, a significantly better fit could be obtained in the experimental range $(x \geq 8 \mu \mathrm{m})$ with a Gaussian profile as given by (9) multiplied by a constant $\gamma_{x \rightarrow 0}$ between 0 and 1 . The best fit is obtained in this case for $W_{\mathrm{V}}=$ 1.44 (3) $\mu \mathrm{rad}$ and $\gamma_{x \rightarrow 0}=0.924$ (25).

\section{Discussion}

For the one-dimensional phase grating, we have recorded images at $D=D_{\mathrm{R}} / 2$ and $D=$ $3 D_{\mathrm{R}} / 2$ (with the grating lines along the vertical and the horizontal directions separately), so that we could only apply the approach based on the Gaussian model for the source profile to determine $W_{\mathrm{V}}$ and $W_{\mathrm{H}}$. Actually, it is unfortunate that we have omitted to record a set of images close to $D=0$ and around $D=$ $D_{\mathrm{R}}$ for this grating, in order to use also the slope method.

With the two-dimensional binary phase grating, the most reliable results are certainly those corresponding to $m=1$ (second line of Table 1) for which no correction was necessary. The third Fourier coefficient oscillates much faster: this shows up both in the calculated curve and in (4). We have only two points of the $\tilde{I}(D, 3)$ curves as a 

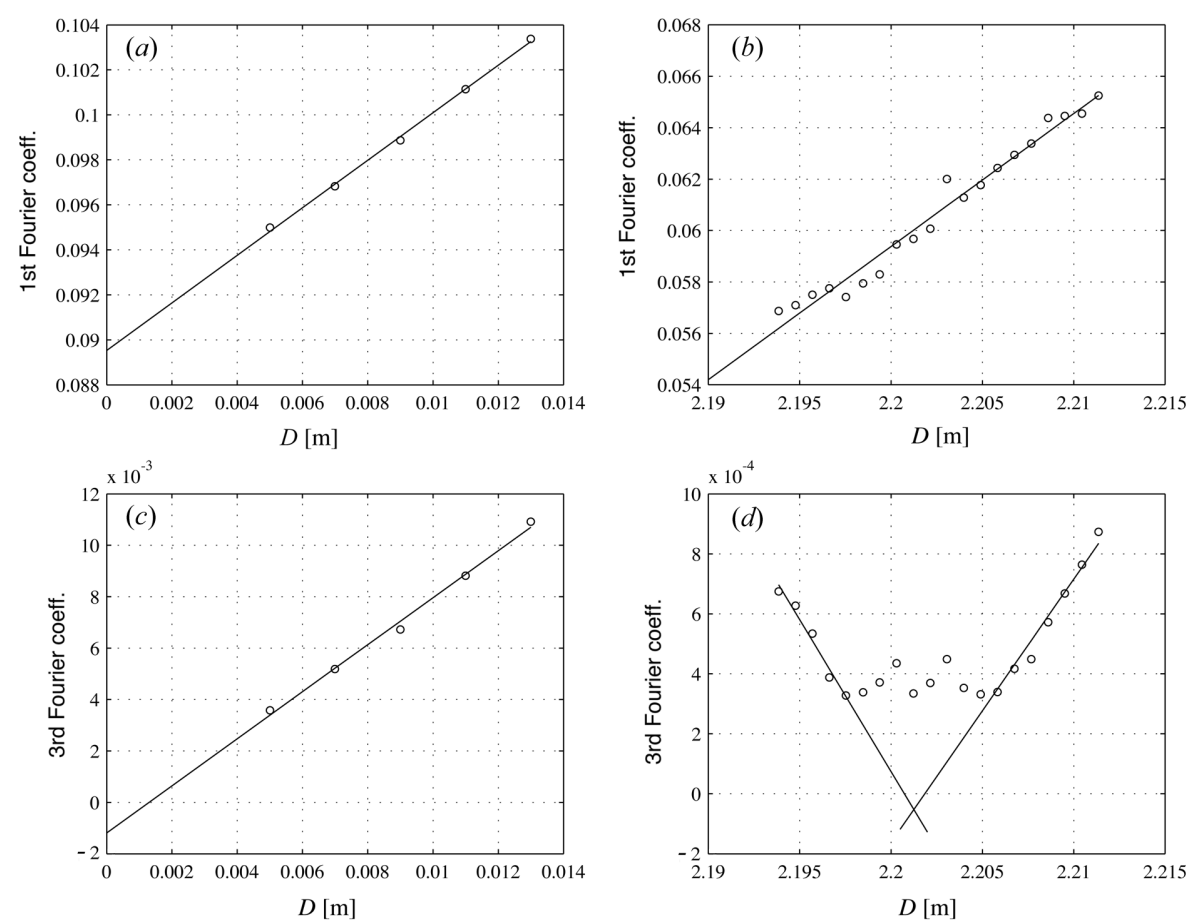

Figure 5

Variation with $D$ of the fundamental $\tilde{I}(D, 1)$ and third $\tilde{I}(D, 3)$ Fourier coefficients of the intensity from the two-dimensional mixed object, in the vertical direction, in the vicinity of $(a),(c) D=0$ and of $(b),(d) D=$ $D_{\mathrm{R}}=2.2 \mathrm{~m}$. The solid lines correspond to linear fits of the measured data.

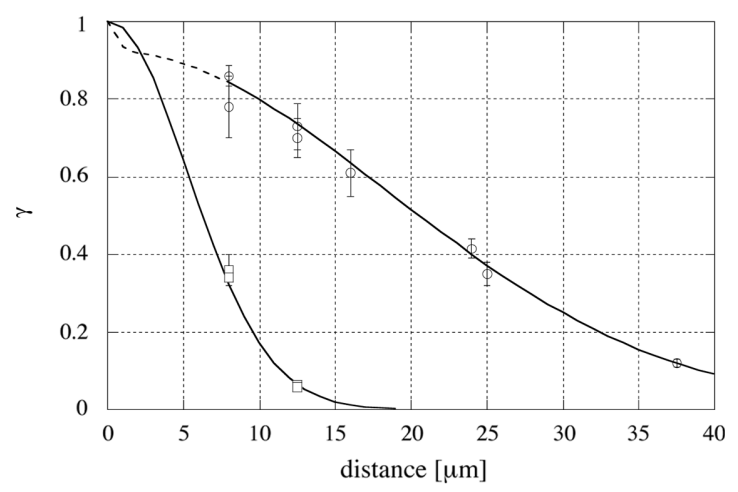

\section{Figure 6}

Measurements of the degree of coherence as a function of distance in the vertical (circular data points) and horizontal (square data points) direction. The full lines are weighted fits of the experimental data points. In the horizontal direction a Gaussian fit with an angular source size $W_{\mathrm{H}}=$ 4.98 (6) $\mu \mathrm{rad}$ is used. In the vertical direction, a Gaussian profile with angular source size $W_{\mathrm{V}}=1.44(3) \mu \mathrm{rad}$ was multiplied with a constant $\gamma_{x \rightarrow 0}=$ $0.924(25)$.

function of $D$ near $D=0$ (at $D=8 \mathrm{~mm}$ and $D=50 \mathrm{~mm}$ ), and two on either side $(D=252 \mathrm{~mm}$ and $D=354 \mathrm{~mm})$ of the partial Talbot position $D_{\mathrm{R} 3}=300 \mathrm{~mm}$. As a result of the scarcity of data, related, as mentioned above, to the fact that the experiments were performed before the possibilities were fully understood, there is a clear discrepancy between the values of $\gamma_{\mathrm{V}}(x=8 \mu \mathrm{m})$ quoted in the second and third lines of Table 1 . It can be noted that, for this grating with similar profiles along two perpendicular directions, the vertical and horizontal Fourier coefficients should be almost the same for small values of $D$, because the attenuation factor should still be close to unity in both cases. This condition is not as well satisfied by our results for $m=3$ as for $m=1$. We attribute this discrepancy to a deterioration of the spatial resolution in the horizontal direction due to vibrations, the effect of which is more pronounced for the higher harmonics. For the simulated solid lines of Fig. 3, we therefore used different values of the effective detector transfer function in the horizontal and vertical direction.

It is interesting to observe that we find in expression (4) the same $D$-dependent factor $\sin \left(\pi \lambda D f^{2}\right)$ we obtain for a phase object $g(x)=\exp [i \varphi(x)]$ such that $|\varphi(x)|<<1$, or, more precisely, such that $|\Delta \varphi|=\mid \varphi(x)-$ $\varphi(x+\lambda D m / a) \mid<<1$, which allows the 'weak phase approximation' $\exp [i \Delta \varphi(x)] \simeq 1+$ $i \Delta \varphi(x)$ (Guigay, 1977). If the duty cycle of the binary phase grating is exactly equal to $1 / 2$ [this means $w=1 / 2$ in formula (4)], the period of $\tilde{I}(D, m)$ is $D_{\mathrm{R} m} / m$ instead of $D_{\mathrm{R} m}$. This property, which is indeed almost verified on the calculated curves in Fig. 3, would not be satisfied in the case of a phase grating with an arbitrary phase profile; for this reason, we did not use it in the present work.

The measurements using the mixed grating, a gold electron microscopy grid, also lack data in the vicinity of the partial Talbot distance corresponding to the second Fourier coefficient, which has better intensity than the coefficient of order three and which could have been appropriate to measure in an alternative way the degree of coherence for $x=12.5 \mu \mathrm{m}$. The results for this mixed object are nevertheless consistent with those obtained with the other gratings. The possibility of using the image in $D=0$ is the main incentive for working with a mixed grating (a pure amplitude grating is not practical for hard $\mathrm{X}$-rays). The variations of the intensity Fourier coefficients are governed for $D \simeq 0$ and for $D \simeq D_{\mathrm{R} m}$ by a term proportional to $\sin \left(\pi m D / D_{\mathrm{R} m}\right)$ and a term proportional to $\cos \left(\pi m D / D_{\mathrm{R} m}\right)$, the latter being absent in the case of a phase grating. The $\sin \left(\pi m D / D_{\mathrm{R} m}\right)$ term is responsible for the rapid variations of $\tilde{I}(D, m)$ shown in Fig. 5. The extrapolation necessary to estimate $\tilde{I}(D=0, m)$, since it is not possible to record an image for $D=0$, is an obvious factor of uncertainty. Furthermore, the estimation of $\tilde{I}\left(D=D_{\mathrm{R} m}, m\right)$ is sensitive to the uncertainty (about $1 \mathrm{~mm}$ ) in the exact Talbot position. Therefore, a very accurate calibration of the distance $D$ is necessary to measure the ratio of $\tilde{I}(D=0, m)$ for $D=0$ and for $D=D_{\mathrm{R} m}$ in the case of a mixed grating. As shown in Fig. 5 , it is more convenient to measure the slopes, as this can be done with a phase grating.

Actually, we are led to the conclusion that using a pure phase grating and the slope approach will be more suitable than using a mixed grating. We need the values of a function $\tilde{I}(D)$ or of its derivative $\tilde{I}^{\prime}(D)$ for certain values of $D$. It is obviously better if these functions are stationary (almost constant) in the regions of interest. This is realised for $\tilde{I}^{\prime}(D)$ in the case of a phase grating with a symmetric phase profile. Moreover, in the case of hard X-rays, phase gratings are more easily available than absorption gratings.

The horizontal and vertical profiles of the degree of coherence shown in Fig. 6 have, as expected, a Gaussian shape. The source sizes found, 274 (3) $\mu \mathrm{m}$ and 79 (2) $\mu \mathrm{m}$, respectively, in the horizontal and vertical direction agree well with the source sizes expected from the machine parameters $(300 \mu \mathrm{m} \pm 10 \%$ and $87 \mu \mathrm{m} \pm 10 \%$, respectively). In the vertical direction, the Gaussian fit gives $\gamma_{x \rightarrow 0}=0.924$ 
Table 1

Summary of the experimental results.

The first column indicates which of the three gratings was used. The second column gives the approach used. The next columns indicate the order $m$ of the Fourier coefficient used, and the multiple $p$ of the partial Talbot distance $D_{\mathrm{R} m}=a^{2} / m \lambda$ that was taken as the specimen-to-detector distance. The results are expressed either through the value of $\gamma(x=p a)$, the degree of coherence for points a distance $x$ apart in the relevant direction ( $\mathrm{V}$ for vertical, $\mathrm{H}$ for horizontal), or as $W_{\mathrm{V}}$ and $W_{\mathrm{H}}$, the angular source sizes of the X-ray beam in these directions. While $\gamma(x)$ is expected to decrease with increasing $|x|$, the values obtained in all measurements for the angular source size in the vertical and horizontal directions should be consistent.

\begin{tabular}{|c|c|c|c|c|c|c|c|c|}
\hline Grating & Approach & $m$ & $p$ & $x(\mu \mathrm{m})$ & $\gamma_{\mathrm{V}}(x)$ & $W_{\mathrm{V}}(\mu \mathrm{rad})$ & $\gamma_{\mathrm{H}}$ & $W_{\mathrm{H}}(\mu \mathrm{rad})$ \\
\hline $\begin{array}{c}\text { 1D phase grating, } \\
a=6.35 \mu \mathrm{m}\end{array}$ & $\begin{array}{l}r\left(D_{0}, p, m\right) \text { with } \\
\quad D_{0}=D_{\mathrm{R}} / 2=275 \mathrm{~mm}\end{array}$ & 1 & 1 & & & $1.40(14)$ & & $4.90(13)$ \\
\hline \multirow{4}{*}{$\begin{array}{l}\text { 2D binary phase grating, } \\
\qquad a=8.0 \mu \mathrm{m}\end{array}$} & Slopes & 1 & 1 & 8 & $0.860(26)$ & $1.81(18)$ & $0.34(2)$ & $4.87(13)$ \\
\hline & Slopes & 3 & 1 & 8 & $0.78(8)$ & $2.31(51)$ & $0.36(4)$ & $4.74(26)$ \\
\hline & Slopes & 3 & 2 & 16 & $0.61(6)$ & $1.65(17)$ & & \\
\hline & Slopes & 3 & 3 & 24 & $0.415(24)$ & $1.34(14)$ & & \\
\hline \multirow[t]{2}{*}{$\begin{array}{l}\text { 2D binary phase grating, } \\
\quad a=8.0 \mu \mathrm{m}\end{array}$} & $\begin{array}{l}r\left(D_{0}, p, m\right) \text { with } \\
\quad D_{0}=D_{\mathrm{R}} / 2=450 \mathrm{~mm}\end{array}$ & 1 & 1 & & & $1.52(15)$ & & $4.91(13)$ \\
\hline & $\begin{array}{c}r\left(D_{0}, p, m\right) \text { with } \\
D_{0}=150 \mathrm{~mm}\end{array}$ & 3 & 1 & & & $1.34(14)$ & & $4.81(15)$ \\
\hline \multirow{4}{*}{$\begin{array}{l}\text { 2D gold mixed grating, } \\
\quad a=12.5 \mu \mathrm{m}\end{array}$} & $r(0, p, m)$ & 1 & 1 & 12.5 & $0.70(5)$ & $1.79(18)$ & $0.064(6)$ & $4.98(9)$ \\
\hline & $r(0, p, m)$ & 2 & 2 & 25 & $0.35(3)$ & $1.54(6)$ & & \\
\hline & Slopes & 1 & 1 & 12.5 & $0.73(6)$ & $1.68(22)$ & $0.058(7)$ & $5.06(11)$ \\
\hline & Slopes & 3 & 3 & 37.5 & $0.12(1)$ & $1.44(2)$ & & \\
\hline
\end{tabular}

instead of 1 . This result may be interpreted by considering that the angular distribution of the beam can be modelled as a weighted sum of two Gaussian functions instead of a single one. One of them, actually corresponding to $7.6 \%$ of the total beam intensity, would be so broad that the corresponding coherence width in the object plane would be much smaller than $8 \mu \mathrm{m}$, which is the smallest distance $x$ in our measurements of the degree of coherence $\gamma(x)$; thus it would have no effect on our measurements, but would contribute for smaller values of $x$, thus leading to the hypothetical dotted part of $\gamma_{\mathrm{V}}(x)$ in Fig. 6. This 'incoherent' part must be related to one or several optical elements of the beamline (Be windows inhomogeneities, monochromator vibrations etc.) and cannot be attributed to the electron beam in the storage ring acting as the source. The fact that the correction is needed only in the vertical direction points to an imperfection in the monochromator system with vertical diffraction geometry. This finding illustrates the need for methods that precisely characterize the coherence function of the beam, for instance by repeating the present experiments based on the Talbot effect with gratings of smaller periods.

\section{Conclusion}

The coherence of a synchrotron radiation beam, in the hard X-ray part of the spectrum, can be conveniently measured by using images obtained at various distances $D$ from a periodic specimen. The partial Talbot effect, based on the use of higher-order $(m>1)$ Fourier coefficients $\tilde{I}(D, m)$ of the intensity distribution, is shown and is experimentally confirmed to be useful for the measurement of the degree of coherence $\gamma(x)$ of a synchrotron radiation beam. It can be more convenient than the usual Talbot effect, because the latter entails the use of larger specimen-detector distances. The Fourier coefficients $\tilde{I}(D, m)$, plotted as a function of the defocusing distance $D$, provide, through their gradual decrease, a sampling of the mutual coherence function with period $a$ equal to that of the specimen. The zeros in these $\tilde{I}(D, m)$ are sharp in the case of a periodic phase object, and the absence of contrast, in this case, at $D=0$ can be obviated by a shift of attention to the derivative of $\tilde{I}(D, m)$ with respect to $D$. The coherence properties are directly determined from the ratio of Fourier coefficients, without any need for computer simulations. The other straightforward approach to obtain contrast at $D=0$, viz. the use of a mixed object involving an amplitude variation too, is more complicated because the amplitude part leads to a variation with $D$ that is functionally different from the phase contribution.

The present experiments show that the Talbot method can provide quantitative information over a wide range of spatial coherence. It is a convenient alternative to the double-slit experiments (Chang et al., 2000; Paterson et al., 2001; Leitenberger et al., 2001). Basically, the Talbot method takes advantage of the fact that Fresnel diffraction phenomena are greatly simplified in the case of periodic objects. The information obtained is concentrated on the coherence function $\gamma(x)$ for specific values of $x$ which are integral multiples of the grating period. This is obviously favorable in terms of the signal-to-noise ratio. Furthermore, the results do not depend on the shape of the grating unit-function and they are not influenced by the detector transfer function, since we compare the components with the same spatial frequency in different images. This advantage is clearly illustrated by the difficulties encountered when we compare measurements with simulations, as shown in Fig. 3.

Other Fresnel diffraction phenomena, also based on simple experimental set-ups, have been applied to estimating the spatial coherence of a monochromated hard X-ray beam, for instance the visibility of the diffraction fringes from a slit (Lang et al., 1987), an edge (Cloetens et al.,1996) or a fiber (Kohn et al., 1999). The fiber method is certainly more practical for qualitative rather than for quantitative information, because it is then necessary to numerically fit the experimental patterns to calculations with variable coherence characteristics, a procedure which is obviously not so well defined as compared with using a simple formula such as (6) in the Talbot method. This last remark is also valid for recent coherence measurements (Lin et al., 2003) using a specially designed phase mask (URA-uniform redundant array).

The Talbot method has obvious advantages of simplicity, for coherence measurements, over more sophisticated arrangements such as a Fresnel-mirror interferometry system (Fezzaa et al., 1997) and the method based on the visibility of equal-thickness fringes in dynamical Bragg diffraction (Tamasaku \& Ishikawa, 2001). For small angular source sizes, the Talbot method should be more accurate than more elaborate experiments involving scanning the source with a multiple reflection monochromator with very narrow acceptance angle (Hart \& Siddons, 1982). It is also much simpler than the 
coherence experiments based on intensity interferometry (Kunimune et al., 1997; Gluskin et al., 1999; Yabashi et al., 2001).

We thank E. Ziegler for his help during the experiments at the optics beamline, and the European Synchrotron Radiation Facility for synchrotron radiation facilities (proposal MI540).

\section{References}

Arrizon, V. \& Ojeda-Castañeda, J. (1992). J. Opt. Soc. Am. A9, 1801-1806. Arrizon, V. \& Rojo-Velázquez, G. (2001). J. Opt. Soc. Am. A18, 1252-1256.

Born, M. \& Wolf, E. (1999). Principles of Optics. 7th ed. Cambridge University Press.

Chang, C., Naulleau, P., Anderson, E. \& Attwood, D. (2000) Opt. Commun. 182, 25-34.

Cloetens, P. (1999). PhD thesis. Vrije Universiteit Brussel, Belgium.

Cloetens, P., Barrett, R., Baruchel, J., Guigay, J. P. \& Schlenker, M. (1996). J. Phys. D, 29, 133-146.

Cloetens, P., Guigay, J. P., Baruchel, J., De Martino, C. \& Schlenker, M. (1997). Opt. Lett. 22, 1059-1061.

Fezzaa, K., Comin, F., Marchesini, S., Coïsson, R. \& Belakhovsky, M. (1997). J. $X$-ray Sci. Technol. 7, 12-23.

Gluskin, E., Alp, E. E., McNulty, I., Sturhahn, W. \& Sutter, J. (1999). J. Synchrotron Rad. 6, 1065-1066.

Goodman, J. W. (1968). Introduction to Fourier Optics. New York: McGrawHill.

Guigay, J. P. (1971). Opt. Acta, 18, 677-682.
Guigay, J. P. (1977). Optik, 49, 121-125.

Guigay, J. P. (1978). Opt. Commun. 26, 136-138.

Hart, M. \& Siddons, P. (1982). Nucl. Instrum. Methods, 204, 219-221.

Koch, A., Cloetens, P., Ludwig, W., Labiche, J. C. \& Ferrand, B. (2000). Proceedings of the 5th International Conference on Inorganic Scintillators and their Applications SCINT99, Moscow 1999, Lomonosov Moscow State University, pp. 157-166.

Koch, A., Raven, C., Spanne, P. \& Snigirev, A. (1998). J. Opt. Soc. Am. A15, 1940-1951.

Kohn, V., Snigireva, I. \& Snigirev, A. (1999). Phys. Rev. Lett. 85, 2745-2748.

Kunimune, Y., Yoda, Y., Izumi, K., Yabashi, M., Zhang, X. W., Harami, T., Ando, M. \& Kikuta, S. (1997) J. Synchrotron Rad. 4, 199-203.

Lang, A. R., Kowalski, G., Makepeace, A. P. W., Moore, M. \& Clackson, S. G. (1987). J. Phys. D, 20, 541-544.

Leitenberger, W., Kuznetsov, S. M \& Snigirev, A. (2001). Opt. Commun. 191, 91-96.

Lin, J. J. A., Paterson, D., Peele, A. G., McMahon, P. J., Chandler, C. T., Nugent, K. A., Lai, B., Moldovan, N., Cai, Z., Mancini, D. C. \& McNulty, I. (2003). Phys. Rev. Lett. 90, 074801.

Paterson, D., Allman, B. E., McMahon, P. D., Lin, J., Moldovan, N., Nugent, K. A., McNulty, I., Chantler, C. T., Retsch, C. C., Irving, T. H. K. \& Mancini, D. C. (2001). Opt. Commun. 195, 79-84.

Patorski, K. (1989). Progress in Optics, Vol. 27, edited by E. Wolf. Amsterdam: North Holland/Elsevier Science.

Talbot, H. F. (1836). Philos. Mag. 9, 401-407.

Tamasaku, K. \& Ishikawa, T. (2001). Acta Cryst. A57, 197-200.

Testorf, M. \& Ojeda-Castañeda, J. (1996). J. Opt. Soc. Am. A13, 119-125.

Winthrop, J. T. \& Worthington, C. R. (1965). J. Opt. Soc. Am. 55, 373-381.

Yabashi, M., Tamasaku, K. \& Ishikawa, T. (2001). Phys. Rev. Lett. 87, 140801.

Zhou, C., Wang, L. \& Tschudi, T. (1998). Opt. Commun. 147, 224-228. 